\title{
Modélisation morphodynamique des fonds autour des brise-lames
}

\author{
Emmanuel MIGNOT ${ }^{1}$, Jean-Michel TANGUY ${ }^{2}$, Frédéric PONS ${ }^{2}$ \\ 'Stage de fin d'études Université de Compiègne au CETE Méditerranée, \\ emmanuel.mignot@hmg.inpg.fr \\ ${ }^{2}$ CETE Méditerranée BP 37000, 13791 Aix-en-Provence cédex3. \\ tél : $0442247649 \quad$ jean-michel.tanguy@equipement.gouv.fr
}

Résumé

Le littoral sableux du Golfe du Lion subit depuis plusieurs décennies une érosion continue de ses plages, ce qui met en danger les infrastructures de la frange littorale. Le but de cette étude est de valider le système de modélisation morphodynamique consistant en le couplage de 3 modèles numériques: agitation de la houle, hydrodynamique et évolution des fonds afin d'étudier l'impact des ouvrages sur les évolutions sédimentaires des plages de sable, avec comme cadre d'étude un briselames. La validation de ce système de modélisation est basée sur des essais physiques réalisés au LCHF, complétée par une étude de sensibilité des paramètres principaux intervenant dans les différents codes de calcul.

\begin{abstract}
The sandy coast of the Golfe du Lion has been facing for several decades a continuous erosion of its beaches, endangering the structures along the coast. The purpose of this study is to validate a system of morphodynamics modeling consisting on the coupling of 3 models: wave, hydrodynamics and sea bed evolution to study the impact of structures on sandy beaches evolutions; for example a beach protected by a breakwater. The validation of this morphodynamics system is based on physical studies performed by LCHF, completed by a sensitivity analysis in order to identify and evaluate the main parameters characterizing each code of calculation.
\end{abstract}

\section{Introduction}

L'amélioration des connaissances sur la physique des processus et en informatique a rendu possible l'élaboration d'outils de simulation qui peuvent avantageusement être utilisés comme des outils d'aide à la décision.

Le but de cette étude est de valider le système de modélisation morphodynamique consistant en le couplage de 3 modèles (houle, courant, sédiments) afin d'étudier l'impact d'ouvrages sur les évolutions sédimentaires des plages de sable. Nous avons 
choisi de prendre comme cadre d'étude les mouvements sédimentaires autour d'un brise-lames basé sur des essais physiques réalisés au LCHF.

\section{Essais sur modèle physique}

En 1987 le CETMEF a confié au LCHF une étude sur modèle réduit [3] dans le but d'examiner, l'influence d'ouvrages tels que des brise-lames et des épis sur le trait de côte. La présente étude s'est focalisée sur l'étude des brise-lames en Méditerranée.

\subsection{Conditions expérimentales}

Le modèle physique comprend un générateur de houle à direction variable. Les conditions de houle sont basées sur une représentation statistique annuelle du climat de houle méditerranéen composée de 8 classes (de hauteur allant de 1.5 à $7 \mathrm{~m}$ et de période de 6 à $12 \mathrm{~s}$ ) et représentée sur le graphique suivant. Dans tous les essais physiques, une structure de type tombolo a été obtenue.

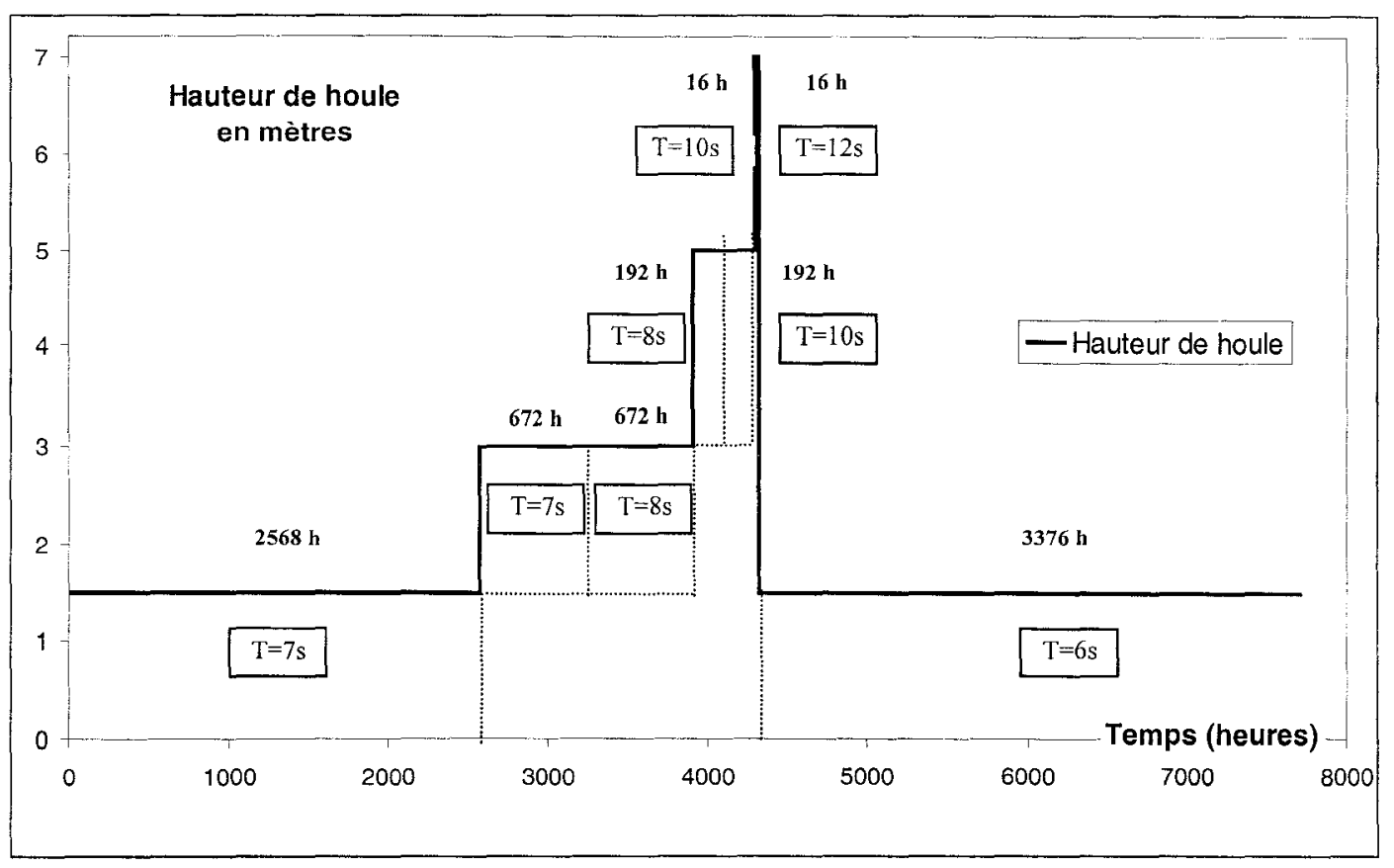

Fig 1 : Climat de houle/wave climate

\subsection{Récapitulation des résultats}

Les mesures prises sur le modèle physique qui ont servi de référence au modèle numérique ont été les hauteurs de houle dans la zone protégée du brise-lames ainsi que les volumes de dépôt accumulés à l'arrière de l'ouvrage. 


\begin{tabular}{|c|l|l|}
\hline $\begin{array}{c}\text { Distance à } \\
\text { la côte } \mathrm{D}\end{array}$ & \multicolumn{2}{|c|}{$\begin{array}{c}\text { Longueur brise-lames } \mathrm{L} \\
100 \mathrm{~m}\end{array}$} \\
\hline $100 \mathrm{~m}$ & & $150 \mathrm{~m}$ \\
\hline $150 \mathrm{~m}$ & $\begin{array}{l}4000 \\
4000(2)\end{array}$ & 10000 \\
\hline $200 \mathrm{~m}$ & & $\begin{array}{l}6000 \\
17000(2)\end{array}$ \\
\hline
\end{tabular}

Ce tableau donne les valeurs approchées des volumes de tombolo accumulés $\left(\mathrm{en}^{3}\right)$ sur un cycle annuel.

(1) Ouvrage semi submersible

(2) Evolution après 2 cycles annuels

Fig 2 Résultats modèle physique Physical results

Un essai prolongé sur 2 cycles a montré que l'équilibre du tombolo était plus rapidement atteint avec un ouvrage court. En effet, un ouvrage plus long permet au tombolo de s'accentuer au cours d'un $2^{\text {ème }}$ cycle, tant en volume qu'en épaisseur. Le temps nécessaire pour obtenir l'équilibre est donc lié aux caractéristiques de l'ouvrage et en particulier au rapport $\mathrm{D} / \mathrm{L}$.

\subsection{Influence de la distance à la côte et de la longueur de l'ouvrage}

Les essais physiques sont lancés pour différentes distances d'implantation du briselames à la côte "D" et longueurs de brise-lames " $L$ ".

- Influence de la distance à la côte "D": En première année, le volume du tombolo formé derrière le brise-lames ainsi que les érosions à ses côtés sont d'autant plus importants que l'ouvrage est proche de la côte. Après la deuxième année, les évolutions sédimentaires ont eu le temps de se stabiliser et cette tendance s'inverse

- Influence de la longueur du brise-lames "L": Deux ouvrages de longueurs différentes, implantés à une même distance de la côte ont été expérimentés. Il en ressort que le volume de sable déposé est plus important avec un ouvrage long.

\section{Simulation numérique}

\subsection{Les modèles utilisés}

La modélisation des phénomènes étudiés sur modèle physique est effectuée à partir de trois modèles couplés (fig.3) :

Le modèle d'agitation REFONDE est basé sur l'équation linéaire de Berkhoff qu'il résout par la méthode des éléments finis (maillage T3, par longueur d'onde). Il permet de traiter des houles multidirectionnelles et multifréquences.

REFLUX : logiciel hydrodynamique utilisé en conditions stationnaires prenant en compte les contraintes internes de radiation de la houle. Il n'y a pas de rétroaction du modèle de courant sur le modèle de houle.

SISYPHE est un code bidimensionnel en éléments finis simulant le transport solide saturé de sédiment par charriage ou transport total, et les évolutions morphodynamiques associées à partir des caractéristiques hydrodynamiques (houle+courant). La formule de transport de Bijker (1968) est utilisée. 


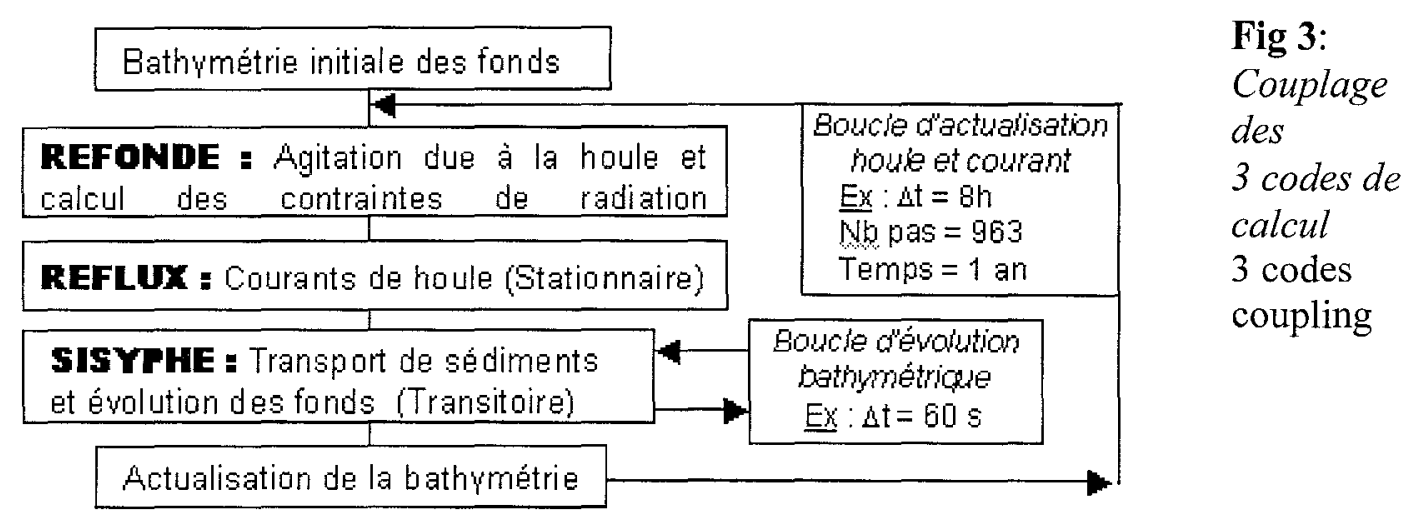

\subsection{Description du cas de référence}

Cette étude a été menée sur le cas suivant :

$\mathrm{D}=150 \mathrm{~m} . \mathrm{L}=100 \mathrm{~m}$. Pente des fonds $=1,4 \%$, Diamètre des grains $=250$ microns.

Maillage à 6 nœuds par longueur d'onde pour Refonde : 16097 nœuds \& 31474 éléments T3. Pour l'hydrodynamique et la morphodynamique, maillage par zone de 8686 éléments T6 pour Reflux et T3 pour Sisyphe. Seul un demidomaine est modélisé du fait de la symétrie du problème. Les conditions limites imposées sont des bords fermés pour les contours de l'ouvrage

Fig 4: Domaine d'étudel domain et la plage .

Les données d'entrée sont les conditions de houle et un niveau d'eau nul au large.

\subsection{Etude de sensibilité des paramètres}

Il a été jugé opportun de mener à bien une étude de sensibilité sur certains paramètres afin d'identifier les plus représentatifs, d'en connaitre les influences réelles et d'en déterminer les valeurs idéales pour la suite de l'étude. Le principal critère de validation est le volume de dépôt à l'arrière de brise-lames.

Viscosité numérique: une augmentation de sa valeur entraîne une diminution des dépôts sédimentaires. La valeur de $40 \mathrm{~m}^{2} / \mathrm{s}$ a été retenue.

Coefficient de rugosité des fonds: il n'a que peu d'influence sur le volume du tombolo, la valeur retenue est le coefficient de Manning, soit $0,025 \mathrm{~m}^{-1 / 3} \mathrm{~s}$.

Coefficient de réflexion à l'arrière du brise-lames: il n'a que peu d'influence sur les volumes déposés. La valeur retenue est 0 .

Coefficient de réflexion à l'avant du brise-lames: il augmente l'agitation à l'avant de ce dernier et augmente le volume de sédiment déposé. La valeur retenue est 0. 
Coefficient de réflexion de la plage: une augmentation de ce coefficient produit une augmentation du volume déposé due à une agitation plus importante en faibles profondeurs. La valeur 0 a été retenue.

L'augmentation du diamètre des grains conduit à une mise en mouvement du sédiment moins importante, donc à un transport sédimentaire plus faible et provoque ainsi une diminution du volume déposé.

Fig 5: Evolution des fonds après Ian.

Bottom evolution after 1 year
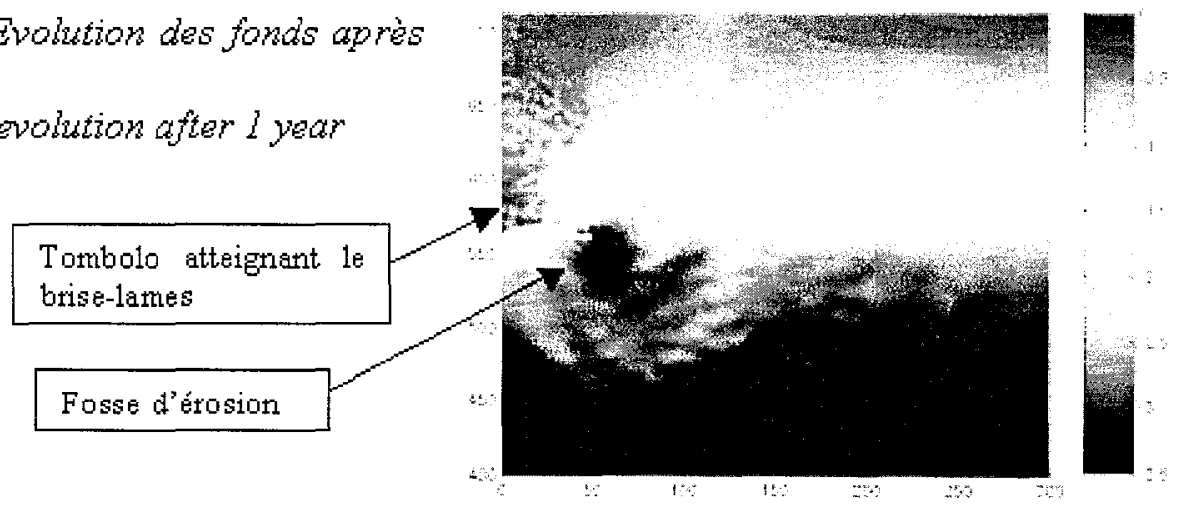

\subsection{Etude de la houle aléatoire}

En complément de cette analyse de sensibilité effectuée sur des houles régulières, (frontales à l'ouvrage), l'étude a été poursuivie en houle aléatoire en utilisant une approche spectrale dite de Jonswap simplifiée (ou de Moskowitz).

On remarque que sur l'ensemble du domaine la hauteur de la houle aléatoire est sensiblement plus faible que celle de la houle régulière qui produit une agitation trop importante. La comparaison entre les résultats du modèle physique et les résultats numériques nous a conduit à retenir une houle aléatoire.

\section{Comparaison avec les connaissances empiriques}

Une fois établi un jeu de valeurs acceptables des paramètres, il s'avère intéressant d'étudier le comportement du code par rapport à des connaissances empiriques qualitatives et quantitatives.

\subsection{Comparaison avec des formules empiriques}

$\checkmark$ Pour ce qui concerne le type de dépôt (tombolo ou salient) que l'on peut s'attendre à obtenir, nos résultats ont été comparés avec [Dally et Pope (1986), Hanson et Kraus (1990), Herbich (1989), Hsu et Silverster (1990), Rosati et al. (1992), Suh et Dalrymple (1987), Sunamura et Mizuno (1987)].

Il est intéressant de noter la disparité des résultats obtenus pour les différentes formules, notamment pour le critère $\mathrm{D} / \mathrm{L}$. Les réponses les plus proches de nos calculs sont celles de Rosati, de Hanson et Kraus et de Sunamura et Mizuno. En revanche, pour Dally et Pope il existe une zone d'indétermination pour $0.5<\mathrm{D} / \mathrm{L}<1.5$ dans laquelle justement se situent nos brise-lames.

La prédiction (brise-lames ou salient) de ces formules sur nos cas est de $70 \%$. 


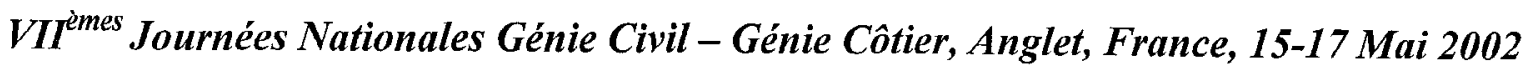

$\checkmark$ Pour ce qui est des ordres de grandeur des volumes déposés à l'arrière de l'ouvrage, les formules sont de [Harris et Herbich (1986), Herbich (1989), Nir (1982)]. Il apparaît là aussi des disparités dans les prévisions, mais globalement nos résultats et ceux des essais physiques sont dans la moyenne de ces formules.

\subsection{Analyse comportementale}

La formule de déferlement utilisée dans le code est la formule de Miche.

Il apparaît que les houles faibles et moyennes déferlent à l'arrière de l'ouvrage, alors que les houles fortes déferlent à l'avant.

\section{- Courantologie au voisinage du brise-lames de référence}

Les visualisations des vitesses des courants de houle calculés par REFLUX permettent de vérifier leur concordance avec les connaissances empiriques.

On montre notamment que:

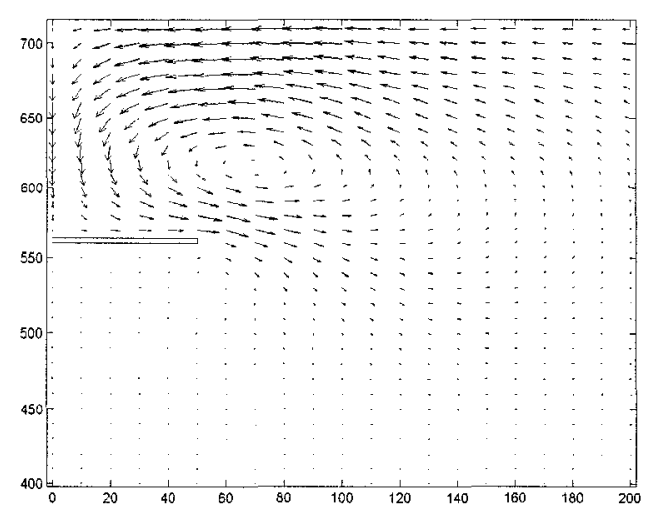

- Les courants importants ont lieu dans la zone de déferlement, où l'on rencontre de fortes contraintes de radiation.

- On retrouve le courant en spirale appelé courant primaire de la zone de déferlement dû à la diffraction des houles en présence d'un ouvrage. Il se situe bien sur le côté du brise-lames, un peu en retrait, correspondant au lieu d'érosion de la fosse.

Fig 6: vecteurs vitesse Velocity vectors

- Etude sédimentologique sur le brise-lames de référence

Les comportements spatiaux observés expliquent l'impact des différents climats de houle sur la formation du tombolo ( $1^{\text {ere }}$ année) et sur son évolution ( $2^{\text {ème }}$ année).

Année $n^{\circ} 1$ : période de formation du tombolo

Lors des faibles houles, le tombolo se forme et progresse vers le brise-lames à partir des sédiments provenant de la fosse en cours de formation.

Pour les houles moyennes $(H=3 \mathrm{~m})$, le processus se poursuit, avec un certain « lissage ». Les dépôts rejoignent l'ouvrage et la fosse continue de s'approfondir.

Les périodes de tempête, caractérisées par de fortes houles, provoquent un léger recul $\mathrm{du}$ tombolo dans sa partie proche de la fosse, compensée par une avancée et un épaississement suivant son axe. Les sédiments érodés remplissent partiellement la partie de la fosse proche de celui-ci, les fonds devant l'ouvrage continuent de s'approfondir.

La période de retour au calme provoque une avancée décisive du tombolo, qui s'épaissit au niveau de l'ouvrage, la plage s'érode du fait des courants de recirculation et approvisionne le tombolo, la fosse d'érosion migre vers le large. 
Année $n^{\circ} 2$ : évolution annuelle à l'équilibre

L'année 2 débute par une période de calme qui a tendance à stabiliser le tombolo et à combler la fosse d'érosion.

La période de tempête, similaire à celle de la $1^{\text {ère }}$ année, provoque un recul du tombolo, accompagné d'un engraissement de la plage. La période de calme prvoque un recul la plage et un engraissement du tombolo. La fosse d'érosion réapparait et il se produit une avancée des fonds devant le brise-lames vers le large.

Ainsi en période de houle moyenne, une fosse d'érosion se forme en tête de l'ouvrage. Elle a tendance à alimenter le tombolo.

Les tempêtes ont tendance à répartir les matériaux sur le domaine, diminuant le volume du tombolo et comblant la fosse d'érosion.

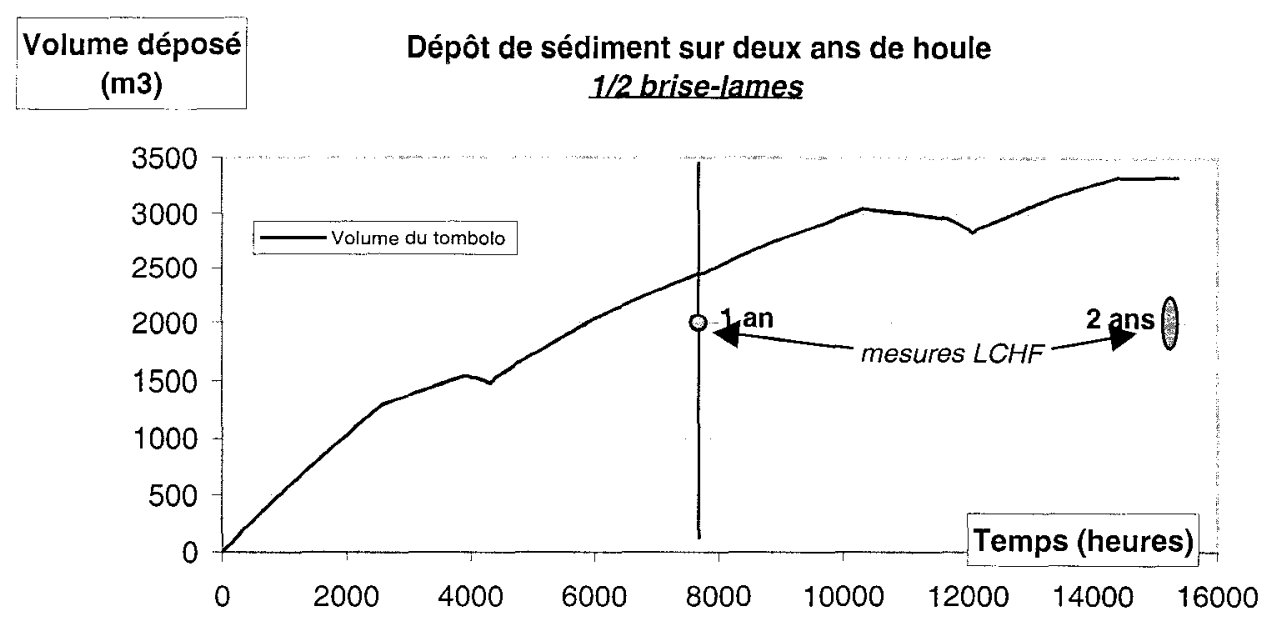

Fig. 7 : dépôts de sédiments à l'arrière du brise-lames / Deposition volume in the lee of the breakwater

Le tombolo de référence atteint l'équilibre après 2 ans avec une faible courbe de progrẹssion du volume de dépôt sur la deuxième année.

- Etude sédimentologique pour différents brise-lames

Nous avons ensuite repris les configurations physiques étudiées par le LCHF avec le même cycle de houle annuel et avons conduit les simulations pour ces brise-lames afin de vérifier les influences des deux paramètres d'implantation: longueur et distance à la côte des ouvrages.

\begin{tabular}{|l|l|l|l|}
\hline $\mathbf{D}(\mathbf{m})$ & $\mathbf{L}(\mathbf{m})$ & $\begin{array}{l}\text { Essais physiques } \\
\left(\mathbf{m}^{\mathbf{3}}\right) \text { demi-BL }\end{array}$ & $\begin{array}{l}\text { Résultats numériques } \\
\left(\mathbf{m}^{\mathbf{3}}\right)\end{array}$ \\
\hline 150 & 100 & 2000 & 2446 \\
\hline 200 & 150 & $3000(7500=2$ ans $)$ & 7373 \\
\hline 150 & 150 & 5000 & 3578 \\
\hline 100 & 150 & $500(1)$ & 1059 \\
\hline 500 & 150 & Non étudié & $12897 \quad(2)$ \\
\hline
\end{tabular}

(1) partiellement submersible

(2) formation d'un salient et non d'un tombolo. 
La modélisation numérique confirme que le volume déposé augmente si la distance à la côte ou la longueur du brise-lames augmentent.

Par ailleurs, la zone de recirculation varie énormément suivant les différentes configurations. Pour le brise-lames situé près de la côte, l'ouvrage s'avère très perturbateur et l'on voit apparaître de fortes vitesses à son niveau, alors que pour le brise-lames éloigné, les perturbations sont localisées.

Hormis le brise-lames éloigné de $100 \mathrm{~m}$, les formules empiriques encadrent les résultats physiques et numériques pour les brise-lames de $150 \mathrm{~m}$ de long.

- Evolution de la profondeur de la fosse d'érosion

Pour chaque brise-lames étudié, la fosse d'érosion est située de chaque côté de l'ouvrage et est due à une accélération du courant dans cette zone créée par le «rétrécissement » de la zone de passage du fluide.

Au niveau de son évolution, plusieurs périodes peuvent être distinguées :

o Création et approfondissement en beau temps et houles moyennes.

- Stabilisation, voire léger comblement de la fosse en période de tempête.

- Nouvel approfondissement pendant la dernière période de houles moyennes

On remarque que la profondeur maxi de la fosse d'érosion pour un état sédimentaire stabilisé augmente si D augmente mais n'est pas influencé par L.

\section{Conclusion}

Cette étude nous a permis d'évaluer les capacités de ce couplage en termes de modélisation de phénomènes côtiers.:

- Les processus physiques observés ainsi que les connaissances empiriques des phénomènes étudiés ont été bien reproduits par le système de modélisation numérique.

- Nous avons fait ressortir un jeu de valeurs de paramètres principaux et mis en évidence leur impact sur la sensibilité des résultats.

- Le type de cas étudié permet une utilisation de ce système de modélisation sur une échelle spatiale de l'ordre d'un ou deux kilomètres de côté et d'échelle de temps de l'ordre de l'année.

\section{Bibliographie}

[1] R.G. DEAN, R.G. DALRYMPLE Water wave mechanics for engineers and scientists, 1993.

[2] US Army Corps of Engineers, Coastal Groins and Nearshore Breakwaters, EM 1110-2-1617, 1992.

[3] LCHF Division SOGREAH, Ouvrage de protection d'un littoral sableux, Etude sur modèle réduit à trois dimensions, 1987.

[4] H. HANSON et KRAUS, Shoreline response to a single transitive detached breakwater, 1990.

[5] J.B. HERBICH, Shoreline changes due to offshore breakwaters, 1989. 